\title{
Brain-Derived Neurotrophic Factor Serum Levels and Hippocampal Volume in Mild Cognitive Impairment and Dementia due to Alzheimer Disease
}

\author{
Ericksen Mielle Borbaa, d Juliana Avila Duarte ${ }^{\mathrm{c}}$ Giovana Bristot $^{\mathrm{b}}$ \\ Ellen Scotton ${ }^{b}$ Ana Luiza Camozzato ${ }^{a}$ Márcia Lorena Fagundes Chaves ${ }^{a}$ e \\ ${ }^{a}$ Dementia Clinic, Neurology Service, ${ }^{b}$ Bipolar Disorder Program, Laboratory of Molecular \\ Psychiatry, and ${ }^{\mathrm{C}}$ Radiology Service, Hospital de Clínicas de Porto Alegre (HCPA), d Postgraduate \\ Program in Medical Sciences, School of Medicine, and 'Internal Medicine Department, \\ School of Medicine, Universidade Federal do Rio Grande do Sul (UFRGS), Porto Alegre, Brazil
}

Keywords

Alzheimer disease - Blood biomarker - Brain atrophy · Hippocampus - Mild cognitive impairment

\section{Abstract}

Background/Aims: Hippocampal atrophy is a recognized biomarker of Alzheimer disease (AD) pathology. Serum brain-derived neurotrophic factor (BDNF) reduction has been associated with neurodegeneration. We aimed to evaluate BDNF serum levels and hippocampal volume in clinical AD (dementia and mild cognitive impairment [MCI]). Methods: Participants were 10 patients with $\mathrm{MCI}$ and 13 with dementia due to $A D$ as well as 10 healthy controls. BDNF serum levels were determined by ELISA and volumetric measures with NeuroQuant ${ }^{\circledR}$. Results: MCI and dementia patients presented lower BDNF serum levels than healthy participants; dementia patients presented a smaller hippocampal volume than MCI patients and healthy participants. Discussion: The findings support that the decrease in BDNF might start before the establishment of neuronal injury expressed by the hippocampal reduction.

\section{Introduction}

Alzheimer disease (AD) is associated with hippocampal atrophy and memory decline [1, 2]. Memory impairment is one of the most common symptoms in patients in the early stages of $\mathrm{AD}$; it is characterized by explicit memory deficit, particularly episodic and working 
memory functions [3]. These deficits are reflective of the involvement of the hippocampal formation [4]. Impaired verbal and episodic memory were correlated with reduced volume of the hippocampal formation and entorhinal cortex, which may be reduced by $25 \%$ using volumetric scanning [5]. Atrophy was most marked in the entorhinal cortex and superior temporal gyrus and extended to the anterior cingulate [5].

Recently, there has been a conceptual shift towards the proposal that AD exists before the onset of dementia [6]. Therefore, a clinical phenotype of memory/cognitive impairment without significant functional impairment, called mild cognitive impairment (MCI), was included in the diagnostic criteria of the International Working Group [7] and the National Institute of Aging-Alzheimer's Association (NIA-AA) [6]. The NIA-AA criteria also proposed several biomarkers to support this predementia or prodromal AD diagnosis. Among these biomarkers, structural neuroimaging with atrophy of the medial temporal region as positive diagnostic information was included. Combinations of distinct biomarkers, including hippocampal volumetry, present a good predictive value to differentiate AD from normal aging in patients with MCI [6].

Brain-derived neurotrophic factor (BDNF), a neurotrophin highly expressed in the hippocampus [8], is a vital component of synaptic plasticity and memory formation $[9,10]$. BDNF has been related to hippocampal plasticity $[9,11]$ and its action plays a central role in the mechanism of long-term potentiation - one of the most accepted models for memory formation - resulting in structural synaptic changes $[9,10]$. However, despite all the available information, there is no consensus about the serum BDNF profile in neurodegenerative diseases, particularly AD. While some studies have reported lower BDNF serum levels in AD patients in comparison with healthy subjects [12-14], other studies demonstrated higher BDNF serum levels in AD individuals [15] or no difference between AD and controls [16].

Likewise, studies on cerebrospinal fluid (CSF) BDNF levels in AD and other dementias report conflicting results. Forlenza et al. [17] demonstrated decreased CSF BDNF concentration associated with progression to AD in amnestic MCI patients. Blasko et al. [18] did not find a significant difference in BDNF between AD and controls, and Laske et al. [19] showed lower CSF BDNF concentrations among AD patients in comparison to healthy controls and non-AD dementia patients. Furthermore, a study with healthy older adults showed an association of lower CSF BDNF levels with poorer memory performance and faster cognitive decline [20].

The effect of the BDNF genetic variability or circulating levels of BDNF on memory and hippocampal volume in healthy older adults and in individuals with MCI or early dementia has also been evaluated showing discrepant results. Among individuals with amnestic MCI and high amyloid- $\beta$ accumulation, BDNF Val66Met carriers showed a significant and larger decline in episodic memory and hippocampal volume [21]. A significant correlation between lower circulating BDNF and memory deficit was demonstrated in a cohort with MCI participants [14]. BDNF serum levels and hippocampal volume were found to positively correlate in a study with healthy older adults [22], but this relation was not further observed [23]. Additionally, no significant BDNF effect, in terms of genotype or plasma, in both hippocampal volume and memory was found in 2 large independent middle- and old-age cohorts of cognitively normal and early-stage dementia participants [24]. Finally, aging itself is associated with reduced hippocampal volume, lower levels of serum BDNF, and poorer memory performance [22].

Therefore, the objective of the study was the evaluation of BDNF serum levels and hippocampal volume in individuals with MCI and dementia due to AD, as well as in healthy elderly participants. The relationship between BDNF serum levels and hippocampal volume was also evaluated in the whole sample. 


\section{Methods}

\section{Participants}

The sample was composed of 10 healthy elderly individuals, 10 participants with MCI, and 13 with dementia due to AD. Healthy elderly individuals were community-dwelling residents who were accompanying Internal Medicine patients, were functionally independent, and presented a Mini-Mental State Examination (MMSE) score $>26$, cognitive performance above the cutoffs for this population, and a Clinical Dementia Rating Scale score of 0 . The NIA-AA core clinical criteria for MCI due to AD were used to identify the MCI group: (1) cognitive concern reflecting a change in cognition reported by the patient or informant or clinician (i.e., historical or observed evidence of decline over time); (2) objective evidence of impairment in 1 or more cognitive domains, typically including memory (i.e., formal or bedside testing to establish level of cognitive function in multiple domains); (3) preservation of independence in functional abilities; and (4) not demented. In addition, vascular, traumatic, medical causes of cognitive decline and the autosomal dominant form of AD were ruled out by clinical history [6]. All dementia patients met the NIA-AA criteria for probable dementia due to AD [25]. Patients (MCI and dementia) were selected from the Dementia Outpatient Clinic of the Hospital de Clínicas de Porto Alegre, Porto Alegre, Brazil.

All subjects underwent a clinical history and physical examination, the MMSE [26, 27], the Clinical Dementia Rating Scale [28-30], the Activities of Daily Living and Instrumental Activities of Daily Living functional scales [31] and a multidomain cognitive evaluation. This cognitive battery assessed episodic memory, executive function, attentional control, visuospatial skills and language by the Rey Auditory Verbal Learning Test [32], the figure copying and the CERAD word list test [33], the clock-drawing test [34], the Trail Making Test [35], the digit span forward [36], and the letter and category fluency [37]. The available age and educational norms were considered to evaluate the scores of each test.

All participants and/or a proxy gave written informed consent and all procedures were approved by the Hospital de Clínicas de Porto Alegre Research Ethics Committee (\# 110275).

\section{BDNF Measurement}

Blood nonfasting samples were collected in serum-separating tubes during clinical evaluations, centrifuged, aliquoted, and stored at $-80^{\circ} \mathrm{C}$.

BDNF levels in serum samples were determined by sandwich ELISA using monoclonal antibodies specific for BDNF (R\&D Systems, Minneapolis, MN, USA). Briefly, microtiter plates were coated overnight at room temperature with the monoclonal anti-BDNF antibody at $4 \mu \mathrm{g} /$ $\mathrm{mL}$ in PBS. Then, plates were washed 3 times with wash buffer and blocked for $1 \mathrm{~h}$ at room temperature with PBS containing 5\% nonfat milk powder. After washing, plates were coated overnight at $4{ }^{\circ} \mathrm{C}$ with the samples diluted 1:200 in sample diluent (PBS with 1\% BSA) and the standard curve ranged from 7.8 to $500 \mathrm{pg} / \mathrm{mL}$ of BDNF. Plates were washed again and biotinylated anti-BDNF antibody at $0.2 \mu \mathrm{g} / \mathrm{mL}$ in PBS was added, which was incubated for $2 \mathrm{~h}$ at room temperature. After washing, incubation with streptavidin-peroxidase conjugate (diluted 1:1,000 in sample diluent) for $1 \mathrm{~h}$ at room temperature was performed. Plates were washed and incubated with the substrate for $20 \mathrm{~min}$ at room temperature. Finally, the stop solution was added and the amount of BDNF was determined by absorbance at $450 \mathrm{~nm}$. The standard curve demonstrated a direct relationship between optical density and BDNF concentration.

\section{Magnetic Resonance Imaging Acquisition}

Magnetic resonance imaging (MRI) data were obtained in a Philips Achieva 1.5-Tesla scanner (Amsterdam, the Netherlands). T1 high-resolution sagittal 3D magnetizationprepared rapid acquisition gradient echo was acquired with NEX $=1$, image matrix $=256 \times$ 
Table 1. Demographic and clinical data of the studied groups

\begin{tabular}{lcccc}
\hline Variables & $\begin{array}{l}\text { Healthy participants } \\
(n=10)\end{array}$ & $\begin{array}{l}\text { MCI } \\
(n=10)\end{array}$ & $\begin{array}{l}\text { Dementia } \\
(n=13)\end{array}$ & $p$ value \\
\hline Age, years $^{\mathrm{a}}$ & $68.60 \pm 4.62$ & $69.20 \pm 7.88$ & $76.08 \pm 6.08^{*}$ & 0.012 \\
${\text { Female, } \mathrm{n}(\%)^{\mathrm{b}}}_{\text {Education, years }^{\mathrm{a}}}$ & $6(60 \%)$ & $6(60 \%)$ & $10(76.9 \%)$ & 0.602 \\
CDR-SB $^{\mathrm{a}}$ & $0.24 \pm 1.14$ & $9.60 \pm 6.07$ & $6.92 \pm 6.17$ & 0.145 \\
MMSE score $^{\mathrm{a}}$ & $28.85 \pm 1.14^{* *}$ & $1.39 \pm 1.13$ & $7.43 \pm 1.06^{* *}$ & 0.001 \\
\hline
\end{tabular}

CDR-SB, Clinical Dementia Rating Scale Sum of Boxes; MMSE, Mini-Mental State Examination. ${ }^{a}$ One-way ANOVA with Bonferroni post hoc test. ${ }^{b} \chi^{2}$ test with Fisher exact test. ${ }^{*} \mathrm{p}<0.05{ }^{* *} \mathrm{p}<0.001$.

232, flip angle $=8^{\circ}, \mathrm{TE}=4 \mathrm{~ms}, \mathrm{TR}=8.63 \mathrm{~ms}$, and voxel size $=1 \times 1 \times 1 \mathrm{~mm}^{3}$ yielding 160 slices . Axial fluid-attenuated inversion recovery was acquired with TR $=11,000 \mathrm{~ms}$, TE $=140 \mathrm{~ms}$, $\mathrm{TI}=2,800 \mathrm{~ms}$, turbo factor $=55$, EPI factor $=1, \mathrm{NEX}=3$, slice thickness $=5 \mathrm{~mm}$, and matrix $=$ $186 \times 512$ providing pixel measurements of $1 \times 0.86 \mathrm{~mm}$ given the $220 \times 220 \mathrm{~mm}$ field of view. Study time for this version of fluid-attenuated inversion recovery was 2 min and $56 \mathrm{~s}$.

\section{Volumetric Analysis}

We obtained volumetric measurements from the high-resolution anatomic images using NeuroQuant ${ }^{\circledR}$ (CorTechs Labs Inc., San Diego, CA, USA), which is a fully automated program that has been validated against other segmentation procedures and found sensitivity to volumetric changes in mild AD [38] and MCI [39]. This program provides volumes for 2 medial temporal lobe regions (hippocampus and inferior lateral ventricles). NeuroQuant (automated) analyzes 3D MRI scans offering accurate, consistent, and reproducible measures and segmentations of brain subcortical structures comparing them with standardized values according to age, sex and cranial volume. The NeuroQuant computer-automated analysis routinely provides volume data on 9 brain regions, left and right sides, for a total of 18 volume measurements (http://www.cortechs.net/products/neuroquant.php). For the present study, we only used the hippocampal and the forebrain parenchyma volumes. The volume of the hippocampus was analyzed as the sum of the right and left hippocampus (total volume), as well as the forebrain parenchyma (total volume).

\section{Statistical Analysis}

Statistical analyses were performed using PASW Statistics 18.0 for Windows (SPSS, Chicago, IL, USA). Descriptive statistics (mean, SD and frequency) were calculated for demographic, cognitive and MRI data. The $\chi^{2}$ test with the Fisher exact test were used for categorical variables, and the Spearman nonparametric correlation was also performed. ANCOVA was used for the analyses controlling for age as covariate. The Bonferroni test was used for the post hoc comparisons.

\section{Results}

Demographic and clinical data are presented in Table 1. Dementia patients were significantly older than MCI and healthy subjects. Moreover, they presented lower MMSE and higher Clinical Dementia Rating Scale Sum of Boxes scores. 
Table 2. Age-controlled comparison of BDNF serum levels and MRI volumetric data among studied groups (ANCOVA with Bonferroni post hoc test)

\begin{tabular}{lccccc}
\hline Variables & $\begin{array}{l}\text { Healthy } \\
\text { participants } \\
(n=10)\end{array}$ & $\begin{array}{l}\text { MCI } \\
(n=10)\end{array}$ & $\begin{array}{l}\text { Dementia } \\
(n=13)\end{array}$ & F & $p$ \\
\hline BDNF level, pg/mL & $26.25 \pm 2.90^{*}$ & $20.57 \pm 2.93$ & $14.59 \pm 2.75$ & 3.72 & 0.036 \\
Forebrain parenchyma volume, $\mathrm{cm}^{3}$ & $949.80 \pm 27.22$ & $926.40 \pm 26.86$ & $860.55 \pm 25.21$ & 2.73 & 0.082 \\
Total hippocampal volume, $\mathrm{cm}^{3}$ & $6.48 \pm 0.23$ & $6.29 \pm 0.26$ & $4.91 \pm 0.24^{* *}$ & 9.74 & 0.001 \\
\hline
\end{tabular}

BDNF, brain-derived neurotrophic factor; $\mathrm{MCI}$, mild cognitive impairment. ${ }^{*} p<0.05$; ${ }^{*} p<0.001$.

Table 2 shows the comparison of BDNF serum levels, hippocampal and forebrain parenchyma volumes among the 3 groups. Healthy participants had higher BDNF levels $(p<0.001)$ than dementia and $\mathrm{MCI}$ patients. The dementia group presented a significantly lower total hippocampal volume than MCI and healthy participants $(p<0.001)$, while the 2 latter groups did not differ. No significant difference in the total forebrain parenchyma volume $\left(\mathrm{cm}^{3}\right)$ among the groups was observed. All analyses were age-adjusted.

To strengthen the results, the correlation analysis was carried out in the whole sample, allowing higher variability of each variable and a larger sample size. However, no significant correlation of serum BDNF with hippocampus volume was observed (Spearman rho $=-0.04$; $p=0.87)$.

\section{Discussion}

The present study was carried out to evaluate BDNF serum levels and hippocampal volume in healthy elderly participants and in patients with $\mathrm{MCI}$ and dementia due to $\mathrm{AD}$ according to the NIA-AA criteria [6, 25]. We also aimed to analyze the correlation of serum BDNF and hippocampal volume in the whole sample. We have hypothesized a decreasing gradient of BDNF serum levels and hippocampal volume from healthy participants toward $\mathrm{MCI}$ and dementia due to $\mathrm{AD}$, based on the following assumptions: (a) BDNF plays a central role in long-term memory potentiation mechanism $[9,10]$; (b) memory impairment is an early and typically clinical phenotype of AD pathology [6, 7]; (c) episodic memory impairment and hippocampal volume reduction are associated [1-3, 40]; and (d) hippocampal volumetry was included in the set of biomarkers proposed to differentiate AD from normal aging in patients with MCI. We also hypothesized a relation of BDNF levels with hippocampus volume, taking into account the high expression of BDNF in the hippocampus [8] and its relation with hippocampal plasticity $[9,11]$. Our main findings are: (1) healthy participants present significantly higher BDNF serum levels than patients with MCI and dementia due to AD; (2) dementia patients present a significantly smaller hippocampal volume than $\mathrm{MCI}$ and healthy participants; and (3) BDNF serum levels do not correlate with hippocampal volume. Therefore, our prior hypotheses were partially confirmed.

Healthy participants presented higher BDNF serum levels than MCI and dementia patients. Patients with MCI and those with dementia due to AD presented similar levels. Our findings are similar to the group of studies reporting higher BDNF levels in healthy elderly individuals and reduced levels in MCI and AD patients [12-14, 24, 41]. However, there is no consensus on the pattern of serum BDNF in these conditions, because another set of studies reported higher $[15,42]$ or similar BDNF levels $[16,43,44]$ in patients with AD. Nonetheless, 
our findings support the reduction of serum BDNF in neurodegenerative disease, such as MCI and dementia due to AD. In the study of Forlenza et al. [17], reduced CSF BDNF concentrations predicted progression of $\mathrm{MCI}$ to $\mathrm{AD}$, suggesting the involvement of BDNF in the pathophysiology of neurodegenerative changes in AD.

Analyzing brain volumes, we found a higher hippocampal than forebrain reduction among dementia patients, but not among MCI and healthy participants, emphasizing the disproportional atrophy of these regions in mild stages of dementia due to AD (according to the Clinical Dementia Rating Scale Sum of Boxes distribution). The specific hippocampal atrophy in AD has already been encompassed by the NIA-AA diagnostic criteria recommendation as a structural biomarker of early AD pathology and a predictor of progression $[6,25$, 45]. Looking particularly at the MCI findings, no significant difference in hippocampal volume was found as compared to healthy participants. This could be explained by the use of the NIA-AA MCI core clinical criteria in the present investigation, a syndrome defined by clinical, cognitive, and functional criteria. The NIA-AA group reported that, similar to dementia due to $\mathrm{AD}$, other etiologies in addition to the AD pathophysiological process may coexist in an individual who meets the criteria for MCI due to AD [6]. Such heterogeneity could have impacted the progression of neuronal injury, measured by the volume of the hippocampus, in our MCI patients. Although MCI clinical criteria are recommended to be applied in clinical settings, they are different from MCI research criteria which include a set of neuroimaging and CSF biomarkers [6]. Among these biomarkers, hippocampal volumetry is one of them. Then, starting with the clinical identification of cases we cannot expect full biomarker positivity in all MCI patients. Levels of certainty according to the presence and nature of the biomarker findings were also proposed for the final set of criteria for MCI due to AD [6]. Finally, although the automated program used as segmentation procedure for the volumetric measures is valid and sensitive [38,39], automated hippocampal segmentation algorithms need to be validated against the manual segmentation gold standard [46].

It is accepted that reduced BDNF serum levels may correspond to the lack of neurotrophic support due to increased amyloid- $\beta$ accumulation contributing for the progressive degeneration of specific brain regions affected by Alzheimer pathology [19]. Taking our results together (lower BDNF levels in $\mathrm{MCI}$ and dementia due to $\mathrm{AD}$ and smaller hippocampal volume in dementia due to AD), we can hypothesize that the decrease in BDNF may start before the establishment of neuronal injury expressed by the hippocampal reduction.

The BDNF can cross the blood-brain barrier in both directions and BDNF in the blood and in the cerebral cortex showed a positive correlation in animal studies $[47,48]$. The hippocampus is one of the main regions of BDNF expression in the cerebral cortex, supporting the a priori hypothesis of the present study. However, our findings refute this hypothesis since no relationship of BDNF serum levels with hippocampal volume was found. Therefore, serum BDNF did not seem a consistent marker for hippocampal atrophy.

In agreement with our result, another investigation showed BDNF serum levels reflecting some aspects of neuronal integrity, although no significant relationship between BDNF serum concentrations and any hippocampal brain metabolites were observed [49]. Furthermore, we did not analyze the effect of the variation of the BDNF gene (Val66Met substitution) on the hippocampus volume as already explored. However, these studies are not conclusive since some showed an association [50] or no relation of this genetic variability or of the BDNFcirculating levels with the hippocampus volume in healthy and early dementia subjects [24]. An early effect of the reduction of BDNF on hippocampal volume, i.e. neuronal injury, would be another possibility to explain this finding.

The current study has limitations such as the small sample size and the cross-sectional design preventing another data approach as well as the longitudinal assessment of these patients. However, some methodological strengths of the study should be emphasized. The 
Borba et al:: Brain-Derived Neurotrophic Factor Serum Levels and Hippocampa

comparison of BDNF levels and hippocampal volume in the 3 groups was age-adjusted, since age alone can affect the hippocampal volume, serum BDNF, and memory performance [22]. In addition, the use of neuropsychological tests such as the Rey Auditory Verbal Learning test to measure episodic memory impairment (i.e., the ability to acquire and retain new information), which have age and gender norms for the Brazilian population, provided higher reliability in establishing the clinical AD phenotype.

Finally, this is an exploratory investigation that supports the role of reduced BDNF serum levels (easily measurable) in amnesic phenotypes, as well as the established neuronal injury detected by the hippocampal loss in dementia due to AD. The relation of circulating BDNF with hippocampal volume was not observed, but this issue deserves further investigation with larger samples, a longitudinal design and BDNF cerebrospinal measures.

\section{Acknowledgement}

This work was supported by Fundo de Incentivo à Pesquisa e Eventos (FIPE) from Hospital de Clínicas de Porto Alegre (HCPA) and by a doctoral scholarship from Coordenação de Desenvolvimento de Pessoal de Nível Superior (CAPES), Brazil.

\section{Disclosure Statement}

The authors declare that there are no conflicts of interest.

\section{References}

1 Barnes J, Bartlett JW, van de Pol LA, Loy CT, Scahill RI, Frost C, Fox NC: A meta-analysis of hippocampal atrophy rates in Alzheimer's disease. Neurobiol Aging 2009;30:1711-1723.

-2 Jack CR Jr, Bernstein MA, Fox NC, Thompson P, Alexander G, Harvey D, Borowski B, Britson PJ, L Whitwell J, Ward C, Dale AM, Felmlee JP, Gunter JL, Hill DL, Killiany R, Schuff N, Fox-Bosetti S, Lin C, Studholme C, DeCarli CS, Krueger G, Ward HA, Metzger GJ, Scott KT, Mallozzi R, Blezek D, Levy J, Debbins JP, Fleisher AS, Albert M, Green R, Bartzokis G, Glover G, Mugler J, Weiner MW: The Alzheimer's disease neuroimaging initiative (ADNI): MRI methods. J Magn Reson Imaging 2008;27:685-691.

3 Panagyres PK: The contribution of the study of neurodegenerative disorders to the understanding of human memory. QJM 2004;97:555-567.

-4 Fox NC, Warrington EK, Seiffer AL, Agnew SK, Rossor MN: Presymptomatic cognitive deficits in individuals at risk of familial Alzheimer's disease. A longitudinal prospective study. Brain 1998;121:1631-1639.

-5 Killiany RJ, Gomez-Isla T, Moss M, Kikinis R, Sandor T, Jolesz F, Tanzi R, Jones K, Hyman BT, Albert MS: Use of structural magnetic resonance imaging to predict who will get Alzheimer's disease. Ann Neurol 2000;47: 430-439.

6 Albert MS, DeKosky ST, Dickson D, Dubois B, Feldman HH, Fox NC, Gamst A, Holtzman DM, Jagust WJ, Petersen RC, Snyder PJ, Carrillo MC, Thies B, Phelps CH: The diagnosis of mild cognitive impairment due to Alzheimer's disease: recommendations from the National Institute on Aging-Alzheimer's Association workgroups on diagnostic guidelines for Alzheimer's disease. Alzheimers Dement 2011;7:270-279.

7 Dubois B, Albert ML: Amnestic MCI or prodromal Alzheimer's disease? Lancet Neurol 2004;3:246-248.

-8 Murer MG, Yan Q, Raisman-Vozari R: Brain-derived neurotrophic factor in the control human brain, and in Alzheimer's disease and Parkinson's disease. Prog Neurobiol 2001;63:71-124.

-9 Tyler WJ, Alonso M, Bramham CR, Pozzo-Miller LD: From acquisition to consolidation: on the role of brainderived neurotrophic factor signaling in hippocampal-dependent learning. Learn Mem 2002;9:5:224-237.

10 llen SJ, Watson JJ, Dawbarn D: The neurotrophins and their role in Alzheimer's disease. Curr Neuropharmacol 2011;9:559-573.

11 Lu B, Gottschalk W: Modulation of hippocampal synaptic transmission and plasticity by neurotrophins. Prog Brain Res 2000;128:231-241.

12 Forlenza OV, Diniz BS, Teixeira AL, Ojopi EB, Talib LL, Mendonça VA, Izzo G, Gattaz WF: Effect of brain-derived neurotrophic factor Val66Met polymorphism and serum levels on the progression of mild cognitive impairment. World J Biol Psychiatry 2010;11:6:774-780. 
13 Yasutake C, Kuroda K, Yanagawa T, Okamura T, Yoneda H: Serum BDNF, TNF-alpha and IL-1beta levels in dementia patients: comparison between Alzheimer's disease and vascular dementia. Eur Arch Psychiatry Clin Neurosci 2006;256:7:402-406.

14 Yu H, Zhang Z, Shi Y, Bai F, Xie C, Qian Y, Yuan Y, Deng L: Association study of the decreased serum BDNF concentrations in amnestic mild cognitive impairment and the Val66Met polymorphism in Chinese Han. J Clin Psychiatry 2008;69:1104-1111.

-15 Angelucci F, Spalletta G, di Iulio F, Ciaramella A, Salani F, Colantoni L, Varsi AE, Gianni W, Sancesario G, Caltagirone $\mathrm{C}$, Bossù $\mathrm{P}$ : Alzheimer's disease $(\mathrm{AD})$ and mild cognitive impairment $(\mathrm{MCI})$ patients are characterized by increased BDNF serum levels. Curr Alzheimer Res 2010;7:15-20.

16 O’Bryant SE, Hobson V, Hall JR, Waring SC, Chan W, Massman P, Lacritz L, Cullum CM, Diaz-Arrastia R; Texas Alzheimer's Research Consortium: Brain-derived neurotrophic factor levels in Alzheimer's disease. J Alzheimers Dis 2009;17:337-341.

-17 Forlenza OV, Diniz BS, Teixeira AL, Radanovic M, Talib LL, Rocha NP, Gattaz WF: Lower cerebrospinal fluid concentration of brain-derived neurotrophic factor predicts progression from mild cognitive impairment to Alzheimer's disease. Neuromolecular Med 2015;17:326-332.

18 Blasko I, Lederer W, Oberbauer H, Walch T, Kemmler G, Hinterhuber H, Marksteiner J, Humpel C: Measurement of thirteen biological markers in CSF of patients with Alzheimer's disease and other dementias. Dement Geriatr Cogn Disord 2006;21:9-15.

19 Laske C, Stransky E, Leyhe T, Eschweiler GW, Maetzler W, Wittorf A, Soekadar S, Richartz E, Koehler N, Bartels M, Buchkremer G, Schott K: BDNF serum and CSF concentrations in Alzheimer's disease, normal pressure hydrocephalus and healthy controls. J Psychiatr Res 2007;41:387-394.

20 Li G, Peskind ER, Millard SP, Chi P, Sokal I, Yu CE, Bekris LM, Raskind MA, Galasko DR, Montine TJ: Cerebrospinal fluid concentration of brain-derived neurotrophic factor and cognitive function in non-demented subjects. PLoS One 2009;4:e5424.

-21 Lim YY, Villemagne VL, Laws SM, Ames D, Pietrzak RH, Ellis KA, Harrington K, Bourgeat P, Bush AI, Martins RN, Masters CL, Rowe CC, Maruff P; AIBL Research Group: Effect of BDNF Val66Met on memory decline and hippocampal atrophy in prodromal Alzheimer's disease: a preliminary study. PLoS One 2014;9:e86498.

22 Erickson KI, Prakash RS, Voss MW, Chaddock L, Heo S, McLaren M, Pence BD, Martin SA, Vieira VJ, Woods JA, McAuley E, Kramer AF: Brain-derived neurotrophic factor is associated with age-related decline in hippocampal volume. J Neurosci 2010;30:5368-5375.

23 Driscoll I, Martin B, An Y, Maudsley S, Ferrucci L, Mattson MP, Resnick SM: Plasma BDNF is associated with age-related white matter atrophy but not with cognitive function in older, non-demented adults. PLoS One 2012; 7:e35217.

24 Kim A, Fagan AM, Goate AM, Benzinger TL, Morris JC, Head D: Alzheimer's Disease Neuroimaging Initiative. Lack of an association of BDNF Val66Met polymorphism and plasma BDNF with hippocampal volume and memory. Cogn Affect Behav Neurosci 2015;15:625-643.

-25 McKhann GM, Knopman DS, Chertkow H, Hyman BT, Jack CR Jr, Kawas CH, Klunk WE, Koroshetz WJ, Manly JJ, Mayeux R, Mohs RC, Morris JC, Rossor MN, Scheltens P, Carrillo MC, Thies B, Weintraub S, Phelps CH: The diagnosis of dementia due to Alzheimer's disease: recommendations from the National Institute on AgingAlzheimer's Association workgroups on diagnostic guidelines for Alzheimer's disease. Alzheimers Dement 2011;3:263-269.

26 Folstein MF, Folstein SE, McHugh PR: "Mini-mental state". A practical method for grading the cognitive state of patients for the clinician. J Psychiatr Res 1975;12:3:189-198.

27 Brucki SMD, Nitrini R, Caramelli P, Bertolucci PHF, Okamoto IH: Suggestions for utilization of the mini-mental state examination in Brazil. Arq Neuropsiquiatr 2003;61:777-781.

28 Hughes CP, Berg L, Danziger WL, Coben LA, Martin RL: A new clinical scale for the staging of dementia. Br J Psychiatry 1982;140:566-572.

29 Chaves ML, Camozzato AL, Godinho C, Kochhann R, Schuh A, de Almeida VL, Kaye J: Validity of the Clinical Dementia Rating Scale for the detection and staging of dementia in Brazilian patients. Alzheimer Dis Assoc Disord 2007;21:210-217.

-30 O’Bryant SE, Waring SC, Cullum CM, Hall J, Lacritz L, Massman PJ, Lupo PJ, Reisch JS, Doody R; Texas Alzheimer's Research Consortium: Staging dementia using Clinical Dementia Rating Scale Sum of Boxes scores: a Texas Alzheimer's research consortium study. Arch Neurol 2008;65:1091-1095.

-31 Katz S, Ford AB, Moskowitz RW, Jackson BA, Jaffe MW: The index of ADL: a standardized measure of biological and psychosocial function. JAMA 1963;185:914-919.

-32 Malloy-Diniz LF, Lasmar VAP, Gazinelli Lde S, Fuentes D, Salgado JV: The Rey Auditory-Verbal Learning Test: applicability for the Brazilian elderly population. Rev Bras Psiquiatr 2007;29:324-329.

-33 Bertolucci PH, Okmoto IH, Brucki SMD, Siviero MO, Neto JT, Ramos LR: Applicability of the CERAD neuropsychological battery to Brazilian elderly. Arq Neuropsiquiatr 2001;59:532-536.

34 Fonseca RP, Salles JF, Parente MAMP: Instrumento de Avaliação Neuropsicológica Breve Neupsilin. Porto Alegre, Vetor, 2009.

-35 Hamdan AC, Hamdan E: Effects of age and education level on the Trail Making Test in a healthy Brazilian sample. Psychol Neurosci 2009;2:199-203.

36 Nascimento E: Adaptação, validação e normatização do WAIS-III para uma amostra brasileira; in Wechsler D: WAIS III: manual para administração e avaliação. São Paulo, Casa do Psicólogo, 2004. 
37 Fonseca RP, Parente MAMP, Côté H, Ska B, Joanette Y: Bateria Montreal de Avaliação da Comunicação - Bateria MAC. São Paulo, Pró-Fono, 2008.

-38 Brewer JB, Magda S, Airriess C, Smith ME: Fully-automated quantification of regional brain volumes for improved detection of focal atrophy in Alzheimer's disease. Am J Neuroradiol 2009;30:578-580.

-39 England HB, Gillis MM, Hampstead BM: RBANS memory indices are related to medial temporal lobe volumetrics in healthy older adults and those with mild cognitive impairment. Arch Clin Neuropsychol 2014;29: 322-328.

40 Raz N, Ghisletta P, Rodrigue KM, Kennedy KM, Lindenberger U: Trajectories of brain aging in middle-aged and older adults: regional and individual differences. Neuroimage 2010;51:501-511.

-41 Lee JG, Shin BS, You YS, Kim JE, Yoon SW, Jeon DW, Baek JH, Park SW, Kim YH: Decreased serum brain-derived neurotrophic factor levels in elderly Korean with dementia. Psychiatry Investig 2009;6:299-305.

42 Faria MC, Gonçalves GS, Rocha NP, Moraes EN, Bicalho MA, Gualberto Cintra MT, Jardim de Paula J, José Ravic de Miranda LF, Clayton de Souza Ferreira A, Teixeira AL, Gomes KB, Carvalho MD, Sousa LP: Increased plasma levels of BDNF and inflammatory markers in Alzheimer's disease. J Psychiatr Res 2014;53:166-172.

-43 O’Bryant, SE, Hobson VL, Hall JR, Barber RC, Zhang S, Johnson L, Diaz-Arrastia R: Serum brain-derived neurotrophic factor levels are specifically associated with memory performance among Alzheimer's disease cases. Dement Geriatr Cogn Disord 2001;31:31-36.

-44 Nagahara AH, Merrill DA, Coppola G, Tsukada S, Schroeder BE, Shaked GM, Wang L, Blesch A, Kim A, Conner JM, Rockenstein E, Chao MV, Koo EH, Geschwind D, Masliah E, Chiba AA, Tuszynski MH: Neuroprotective effects of brain-derived neurotrophic factor in rodent and primate models of Alzheimer's disease. Nat Med 2009;15,331-337.

-45 Jack CR Jr, Shiung MM, Weigand SD, O’Brien PC, Gunter JL, Boeve BF, Knopman DS, Smith GE, Ivnik RJ, Tangalos EG, Petersen RC: Brain atrophy rates predict subsequent clinical conversion in normal elderly and amnestic MCI. Neurology 2005;65:1227-1231.

-46 Boccardi M, Bocchetta M, Morency FC, Collins DL, Nishikawa M, Ganzola R, Grothe MJ, Wolf D, Redolfi A, Pievani M, Antelmi L, Fellgiebel A, Matsuda H, Teipel S, Duchesne S, Jack CR Jr, Frisoni GB; EADC-ADNI Working Group on the Harmonized Protocol for Manual Hippocampal Segmentation and for the Alzheimer's Disease Neuroimaging Initiative: Training labels for hippocampal segmentation based on the EADC-ADNI harmonized hippocampal protocol. Alzheimers Dement 2015;11:175-183.

47 Poduslo JF, Curran GL: Permeability at the blood-brain and blood nerve barriers of the neurotrophic factors: NGF, CNTF, NT-3, BDNF. Brain Res Mol Brain Res 1996;36:280-286.

48 Pan W, Banks WA, Fasold MB, Bluth J, Kastin AJ: Transport of brain derived neurotrophic factor across the blood-brain barrier. Neuropharmacology 1998;37:1553-1561.

-49 Lang UE, Hellweg R, Seifert F, Schubert F, Gallinat J: Correlation between serum brain-derived neurotrophic factor level and an in vivo marker of cortical integrity. Biol Psychiatry 2007;62:530-535.

50 Bueller JA, Aftab M, Sen S, Gomez-Hassan D, Burmeister M, Zubieta JK: BDNF Val(66)Met allele is associated with reduced hippocampal volume in healthy subjects. Biol Psychiatry 2006;59:812-815. 